TECHNICAL NOTE

\author{
S. Yoshimura \\ M. Koga \\ K. Toyoda \\ T. Mukai \\ B.-H. Hyun \\ M. Naganuma \\ K. Nagatsuka \\ K. Minematsu
}

\section{Frontal Bone Window Improves the Ability of Transcranial Color-Coded Sonography to Visualize the Anterior Cerebral Artery of Asian Patients with Stroke}

SUMMARY: The frontal bone window (FBW) is a promising approach in evaluating the anterior cerebral artery. The goal of the present study was to determine the rates of detection of the basal cerebral arteries by using the FBW alone and a combination of the FBW with the temporal bone window (TBW) in 163 patients. The combined application improved detection rates of $\mathrm{A} 1(58.6 \%$ versus $46.0 \%, P=$ $.001)$ and $A 2(43.6 \%$ versus $6.7 \%, P<.001)$ compared with the TBW alone.
$\mathbf{T}$ ranscranial color-coded sonography (TCCS) is an established tool for evaluating intracranial cerebral arteries because it can identify and focus a sonographic beam on occluded sites. Although a proper bone window for TCCS is essential for detecting intracranial vascular lesions, the acoustic property of the standard approach via the temporal bone window (TBW) is limited in elderly and female patients. ${ }^{1,2}$ In particular, an optimal TBW is difficult to apply in Asians. ${ }^{3,4}$

Improving the detection rate of the anterior cerebral artery (ACA) by TCCS will increase the opportunity for such patients to undergo noninvasive and real-time evaluations at the bedside in general practice. In particular, Japanese patients will receive considerable benefits from an improved TCCS examination because pathologic changes often arise in the intracranial arteries, including the ACA. ${ }^{5,6}$ However, the ACA is more difficult to detect than the middle cerebral artery (MCA) through the TBW. ${ }^{7}$ Studies of a small cohort have indicated that this limitation could be overcome by an approach through the frontal bone window (FBW) to evaluate the postcommunicating segment (A2) of the ACA from a single center. ${ }^{8}$ However, the utility of the FBW for Asian patients with stroke has not been determined. For Asians, TCCS via the FBW and the TBW might be equally disadvantageous.

The present study determined the rate of detecting the

Received August 7, 2008; accepted after revision November 11.

From the Cerebrovascular Division, Department of Medicine, National Cardiovascular Center, Osaka, Japan.

This study was supported in part by grants-in-aid (H2O-Junkanki-Ippan-019) and a Research Grant for Cardiovascular Disease 18C-5 from the Ministry of Health, Labor and Welfare of Japan.

Please address correspondence to Masatoshi Koga, MD, Cerebrovascular Division, Department of Medicine, National Cardiovascular Center, 5-7-1 Fujishiro-dai, Suita, Osaka 565-8565, Japan; e-mail: koga@hsp.ncvc.go.jp

indicates article with supplemental on-line tables.

Indicates open access to non-subscribers at www.ajnr.org

DOI 10.3174/ajnr.A1452 basal cerebral arteries, including the ACA via the FBW alone and via the FBW combined with the TBW in Japanese patients with stroke.

\section{Technique}

A total of 163 consecutive Japanese patients (46 women; mean age, $69.5 \pm 11.4$ years) with cerebrovascular disease were recruited. The detection rates of the main trunk of the MCA (M1), the precommunicating segment (A1) and $\mathrm{A} 2$ of the ACA, the precommunicating (P1) and the postcommunicating (P2) segments of the posterior cerebral artery (PCA) by TCCS through the FBW and TBW were evaluated by using a Sonos 5500 equipped with an S3 transducer (1.0-3.0 MHz; Philips Medical Systems, Tokyo, Japan) or an iE33 with an S5-1 transducer (1.0-5.0 MHz; Philips Medical Systems). Patients were randomly examined with 1 examination from each scanner. The TBW is located upward and forward of the earhole. The FBW consists of the lateral FBW located above the lateral aspect of the eyebrow and the paramedian FBW slightly lateral to the midline of the forehead (Fig 1). The most prominent vascular structure identified via the lateral FBW is A2 with blood flowing toward the probe. The ipsilateral $\mathrm{A} 1$ is color-coded with flow moving away from the probe, and M1 is coded with flow moving toward the probe. $\mathrm{P} 1$ and $\mathrm{P} 2$ are coded with flow moving toward and away, respectively, from the transducer in the lateral frontal insonation plane. Via the paramedian FBW, A1 and A2 are coded with flow toward the probe, and the distal ACA and cortical branches are coded with flow away from the probe.

Because contralateral intracranial arteries were not often detectable via the TBW and FBW in Japanese patients with stroke, only ipsilateral arteries were assessed. The detection of vascular insonation was defined on the basis of the consensus of 2 or more vascular neurologists. According to detection on color-flow imaging and a sufficiently measurable Doppler spectrum, each arterial site was judged as visible. The rates of visualizing a target artery were calculated for both bone windows. Sonographic contrast-enhancing agents were not applied. Categorized variables were compared by using the $\chi^{2}$ tests or Fisher exact test as appropriate. A value of $P<.05$ was considered statistically significant. 

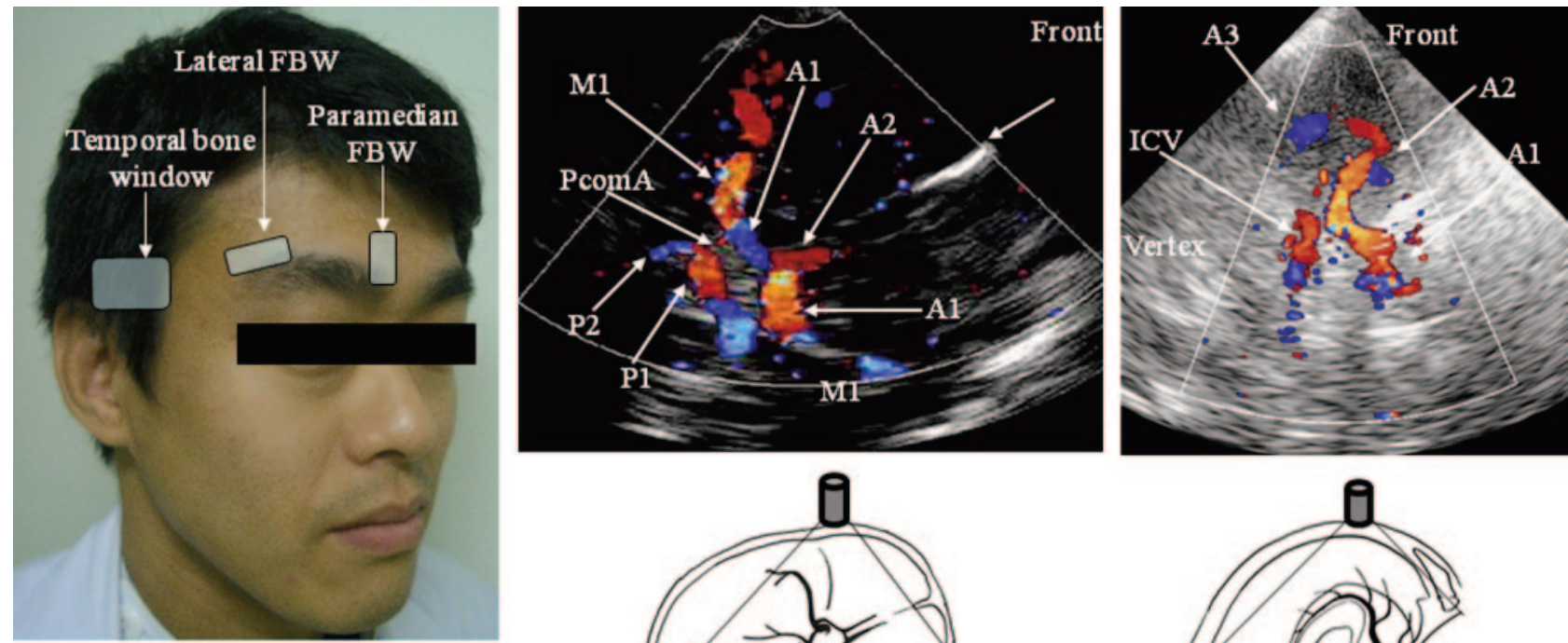

A
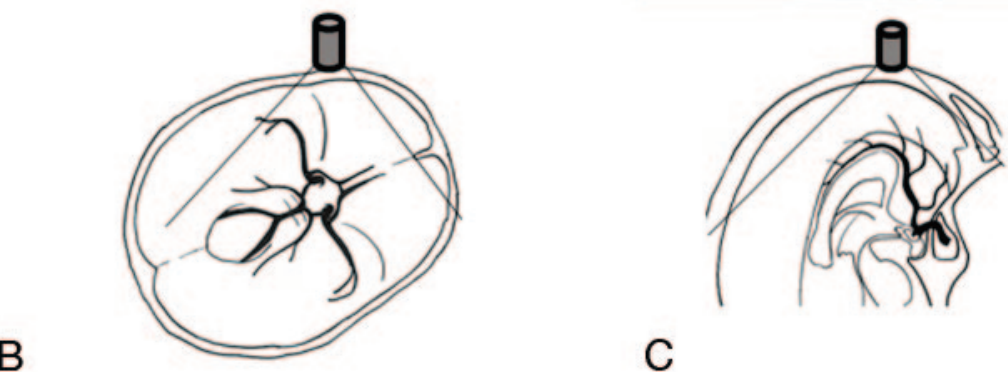

B

C

Fig 1. The lateral and paramedian FBW. ${ }^{8} A$, The lateral FBW is above the lateral aspect the eyebrow. The paramedian FBW is slightly lateral to the midline of the forehead. $B$, TCCS image obtained via the lateral FBW and the schema. M1 and A2 are color-coded with flow moving toward probe. The ipsilateral A1 is coded with flow moving away from probe. P1 and P2 are coded with flow moving toward and away, respectively, from the transducer in the lateral frontal insonation plane. C, TCCS image obtained via the paramedian FBW and the schema. A1 and A2 are color-coded with flow moving toward the probe; and the distal ACA and A3 are color-coded with flow moving away from probe. PcomA indicates posterior communicating artery; ICV, internal cerebral veins,

On-line Table 1 summarizes the rates of visualizing a target artery via the FBW, categorized by age and sex. Detection rates for M1 $(P<$ $.001)$, A1 $(P<.001), \mathrm{A} 2(P<.001), \mathrm{P} 1(P<.001)$, and P2 $(P=.047)$ were always higher in men than in women. The detection rates of $\mathrm{A} 1$ $(P<.001)$ and M1 $(P=.016)$ via the lateral FBW were significantly higher in patients 60 years of age or younger than those older than 60 years of age. On-line Table 2 shows the detection rates of target arteries with TCCS examinations via the TBW and the combination of both windows. The detection rate of A2 was higher via the FBW than via the TBW $(40.2 \%$ versus $6.7 \%, P<.001)$. The combination of the TBW and FBW significantly improved the detection rates of A1 and A2 to $58.6 \%(P=.001)$ and $43.6 \%(P<.001)$ compared with only the TBW (46.0\% and $6.7 \%$, respectively). Only via the FBW, $36.9 \%$ of A2, $12.6 \%$ of $\mathrm{A} 1,4.9 \%$ of $\mathrm{M} 1,6.1 \%$ of $\mathrm{P} 1$, and $2.3 \%$ of $\mathrm{P} 2$ were detected.

\section{Discussion}

This is the first report to describe the clinical utility of TCCS examinations via the FBW in Asian patients with stroke. The rates of detecting all examined arteries were higher in male than in female patients. Approximately $50 \%$ of male and $20 \%$ of female patients with stroke had a proper FBW when the ACA and M1 were visualized. Although the detection rates via the FBW were not better than those via the TBW in all arteries except A2, the combination of the FBW and TBW improved the rate of detecting the ACA. Notably, 36.9\% of A2 and $12.6 \%$ of A1 arteries were detected only via the FBW. The lateral FBW was more useful for the patients without a proper TBW for detecting ACA segments compared with the paramedian FBW.

An improper bone window via the TBW in elderly and female patients is a critical limitation of TCCS examinations. ${ }^{3,4}$ A demonstration of improved rates of ACA visualization by using a combination of the TBW and FBW is important because the insonation quality of Japanese patients with stroke via the TBW is inferior, especially in the ACA territory. Sonographic contrast may be useful for the FBW, to improve detection rates as demonstrated in the TBW.

The present study has some limitations. First, the rates determined in such a small number of female and younger patients with stroke were not statistically reliable. Second, a way to affix the transcranial probe for the FBW for long-term monitoring does not presently exist. Third, the utility of sonographic contrast agents via the FBW was not assessed.

In conclusion, TCCS examination via the FBW combined with the TBW improved the rate of detecting the ACA in Japanese patients with stroke. The compensatory technique appears to be useful for patients with an inadequate TBW.

\section{References}

1. Comerota AJ, Katz ML, Hosking JD, et al. Is transcranial Doppler a worthwhile addition to screening tests for cerebrovascular disease? J Vasc Surg 1995;21: 90-95, discussion 95-97

2. Marinoni M, Ginanneschi A, Forleo P, et al. Technical limits in transcranial Doppler recording: inadequate acoustic windows. Ultrasound Med Biol 1997;23:1275-77

3. Itoh T, Matsumoto M, Handa N, et al. Rate of successful recording of blood flow signals in the middle cerebral artery using transcranial Doppler sonography. Stroke 1993;24:1192-95

4. Yagita Y, Etani H, Handa N, et al. Effect of transcranial Doppler intensity on successful recording in Japanese patients. Ultrasound Med Biol 1996;22:701-05

5. Kazui S, Sawada T, Naritomi H, et al. Angiographic evaluation of brain infarction limited to the anterior cerebral artery territory. Stroke 1993;24:549-53

6. Kameyama M, Okinaka SH. Collateral circulation of the brain with special reference to atherosclerosis of the major cervical and cerebral arteries. $\mathrm{Neu}$ rology 1963;13:279-86

7. Krejza J, Mariak Z, Walecki J, et al. Transcranial color Doppler sonography of basal cerebral arteries in $\mathbf{1 8 2}$ healthy subjects: age and sex variability and normal reference values for blood flow parameters. AJR Am J Roentgenol 1999;172:213-18

8. Stolz E, Kaps M, Kern A, et al. Frontal bone windows for transcranial colorcoded duplex sonography. Stroke 1999;30:814-20 